\title{
An infinite plate with a curvilinear hole having three poles with complex parameters
}

\section{F. S. Bayones ${ }^{1}$ and B. M. Alharbi ${ }^{2}$}

\author{
1,2 Math. Dept., Faculty of Science, Taif University, Saudi Arabia
}

\begin{abstract}
This paper covered the study of the boundary value problem for isotropic homogeneous perforated infinite elastic media. For this, we considered the problem of a thin infinite plate of specific thickness with a curvilinear hole where the origin lie outside the hole is conformally mapped outside a unit circle by means of a specific rational mapping . The complex variable method has been applied and it transforms the problem to the integro-differential equation with Cauchy kernel that can be solved to find two complex potential functions which called Gaursat functions. Many special cases are discussed and established of these functions. Also, many applications and examples are considered. Moreover the components of stress, in each application, are computed.
\end{abstract}

Keywords: Conformal Mapping; Integro-Differential Equation with Discontinuous Kernel; Complex Potential Functions; Cauchey method.

\section{Council for Innovative Research}

Peer Review Research Publishing System

Journal: JOURNAL OF ADVANCES IN MATHEMATICS

Vol .11, No. 5

www.cirjam.com, editorjam@gmail.com 


\section{1- INTRODUCTION :}

Problems dealing with isotropic homogeneous perforated infinite plate have been investigated by several authors. Some author used Laurent's theorem to express each complex potential as a power series [1-4].

Several authors wrote about the boundary value problems and their applications in many different sciences, see Gak-hov [5], Ciarlet et al.[6], Zebib[7] and Saito and Yamamto[8]. From these problems, we established contact problems and mixed problems in the theory of elasticity, see Colton and Kress[9], and Abdou[10]. In Abdou[10,11], Abdou and Khamis[12] and M. A. Abdou and S. J. Monaquel [13], F. S. Bayones and B. M. Alharbi [14] ,Complex variable method is used to express the solutions of these problems in the form of power series by applied Laurant's theorem. Some problems discussed the solution under presence of heat [15-16].

The complex variable method has been applied to solve the first and second fundamental problems for $|\zeta|>1$, the infinite region outside a closed contour conformably mapped outside the unit circle $\gamma$ with two poles . the first and second fundamental problem in the plane theory of elasticity are equivalent to finding analytic functions $\phi_{1}(z)$ and $\psi_{1}(z)$ of one complex argument $z=x+i y$. These functions must satisfy the boundary condition,

$$
K \phi_{1}(t)-t \overline{\phi_{1}^{\prime}(t)}-\overline{\psi_{1}(t)}=f(t)
$$

where $t$ denotes the affix of a point on the boundary. In terms of $z=c w(\zeta), c>0, w^{\prime}(\zeta)$ does not vanish or become infinite for $|\zeta|>1$,

$$
w^{\prime}(\zeta)=0, \infty
$$

where the infinite region is outside a unit circle $\gamma$. For the first fundamental boundary value problems or it called the stress boundary value problems, $K=-1$ and $f(t)$ is a given function of stress. While for $K=k>1, f(t)$ is a given function of displacement which called the thermal conductivity, we have the second fundamental boundary value problems or called the displacement boundary value problems.

The complex potential functions $\phi_{1}(t)$ and $\psi_{1}(t)$ take the following forms, see[17]

$$
\begin{gathered}
\phi_{1}(\zeta)=-\frac{X+i Y}{2 \pi(1+k)} \ln \zeta+c \Gamma \zeta+\phi(\zeta), \\
\psi_{1}(\zeta)=k \frac{(X-i Y)}{2 \pi(1+k)} \ln \zeta+c \Gamma^{*} \zeta+\psi(\zeta),
\end{gathered}
$$

where, $S_{x}, S_{y}$ are the components of the resultant vector of all external forces acting on the boundary and $\Gamma, \Gamma^{*}$ are complex constants. Generally the two complex functions $\phi(\zeta)$ and $\psi(\zeta)$ are single value analytic functions within the region outside the unit circle $\gamma$ and $\phi(\infty)=0, \psi(\infty)=0$.

In this paper, we consider infinite elastic media with a curvilinear hole having three poles and arbitrary shape with complex parameters, in this problem we use the conformal mapping to obtain the complex potential function after rate setting the problem in form of integra-differential with singular kernel. Many special cases are obtain and several applications are discussed form the work .

\section{2- Formulation of the problem :}

Consider the rational mapping on the domain outside a unit circle $\gamma$ by the rational function

$$
z=c \frac{\left(d \zeta+m_{1} \zeta^{-1}+m_{2} \zeta^{-2}+m_{3} \zeta^{-3}\right)}{\left(1-n_{1} \zeta^{-1}\right)\left(1-n_{2} \zeta^{-1}\right)\left(1-n_{3} \zeta^{-1}\right)} \quad, c>0,|\zeta|>1
$$

where $d, m_{j}, n_{j}, j=1,2,3$, are complex parameters,$n_{1} \neq n_{2} \neq n_{3}$

We can written the rational function in the form

$$
z=c \frac{d \zeta^{4}+m_{1} \zeta^{2}+m_{2} \zeta+m_{3}}{\left(\zeta-n_{1}\right)\left(\zeta-n_{2}\right)\left(\zeta-n_{3}\right)}
$$

This mapping must satisfy the condition (2), then 


$$
\begin{aligned}
\frac{z^{\prime}}{c}=\frac{4 d \zeta^{3}+2 m_{1} \zeta+m_{2}}{\left(\zeta-n_{1}\right)\left(\zeta-n_{2}\right)\left(\zeta-n_{3}\right)}-\frac{\left(d \zeta^{4}+m_{1} \zeta^{2}+m_{2} \zeta+m_{3}\right)}{\left(\zeta-n_{1}\right)^{2}\left(\zeta-n_{2}\right)^{2}\left(\zeta-n_{3}\right)^{2}}\left[\zeta-n_{1}\right)\left(\zeta-n_{2}\right) \\
\left.+\left(\zeta-n_{1}\right)\left(\zeta-n_{3}\right)+\left(\zeta-n_{2}\right)\left(\zeta-n_{3}\right)\right] \text { (7) }
\end{aligned}
$$

\section{3- The rational mapping :}

The mapping function (6) maps the curvilinear hole $C$ where the origin lies outside the hole under the conditions that $w^{\prime}(\zeta)$ dose not vanish or become infinite outside the unit circle $\gamma$. The following graphs give the different shapes of the rational mapping (6).
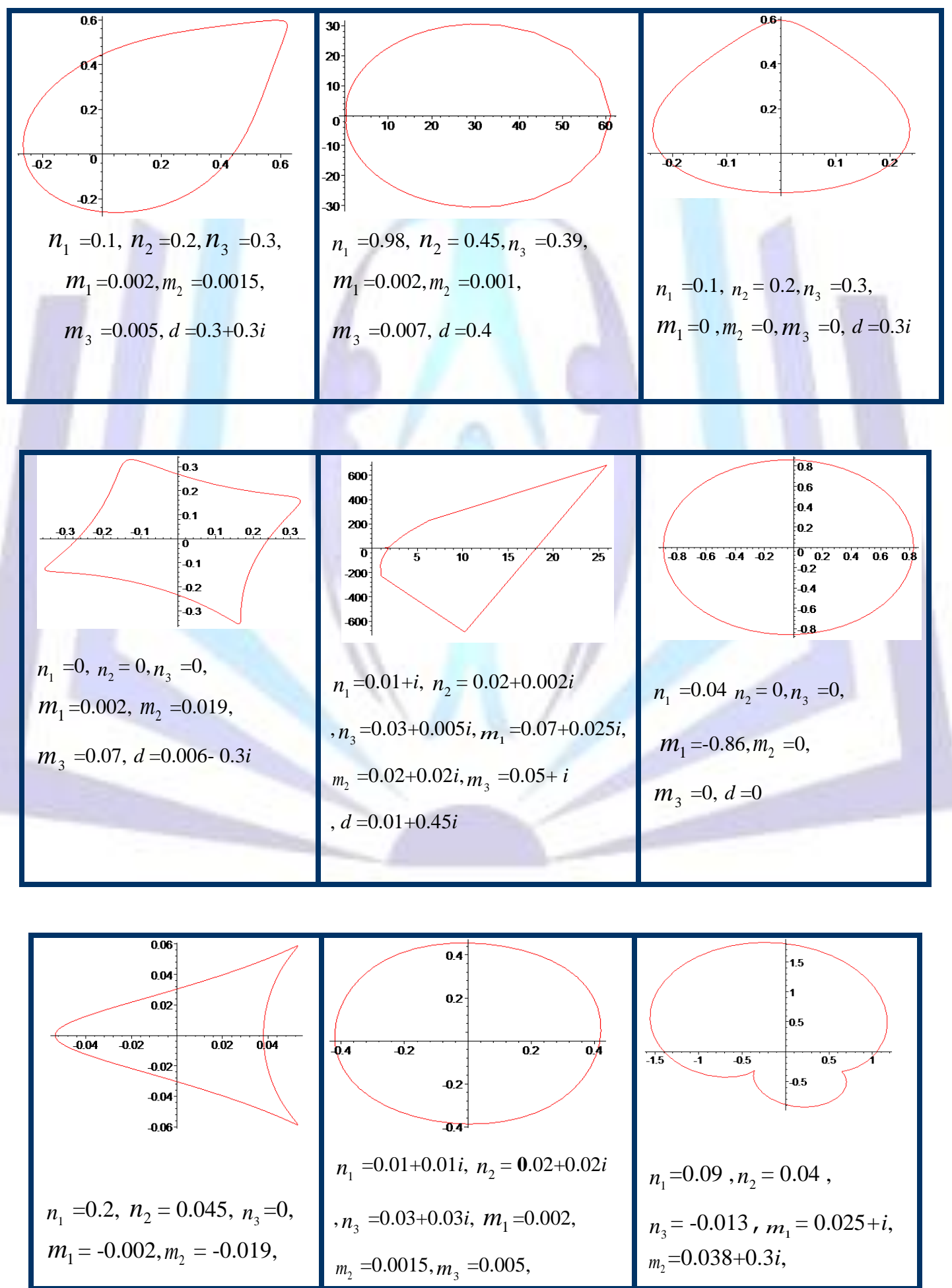

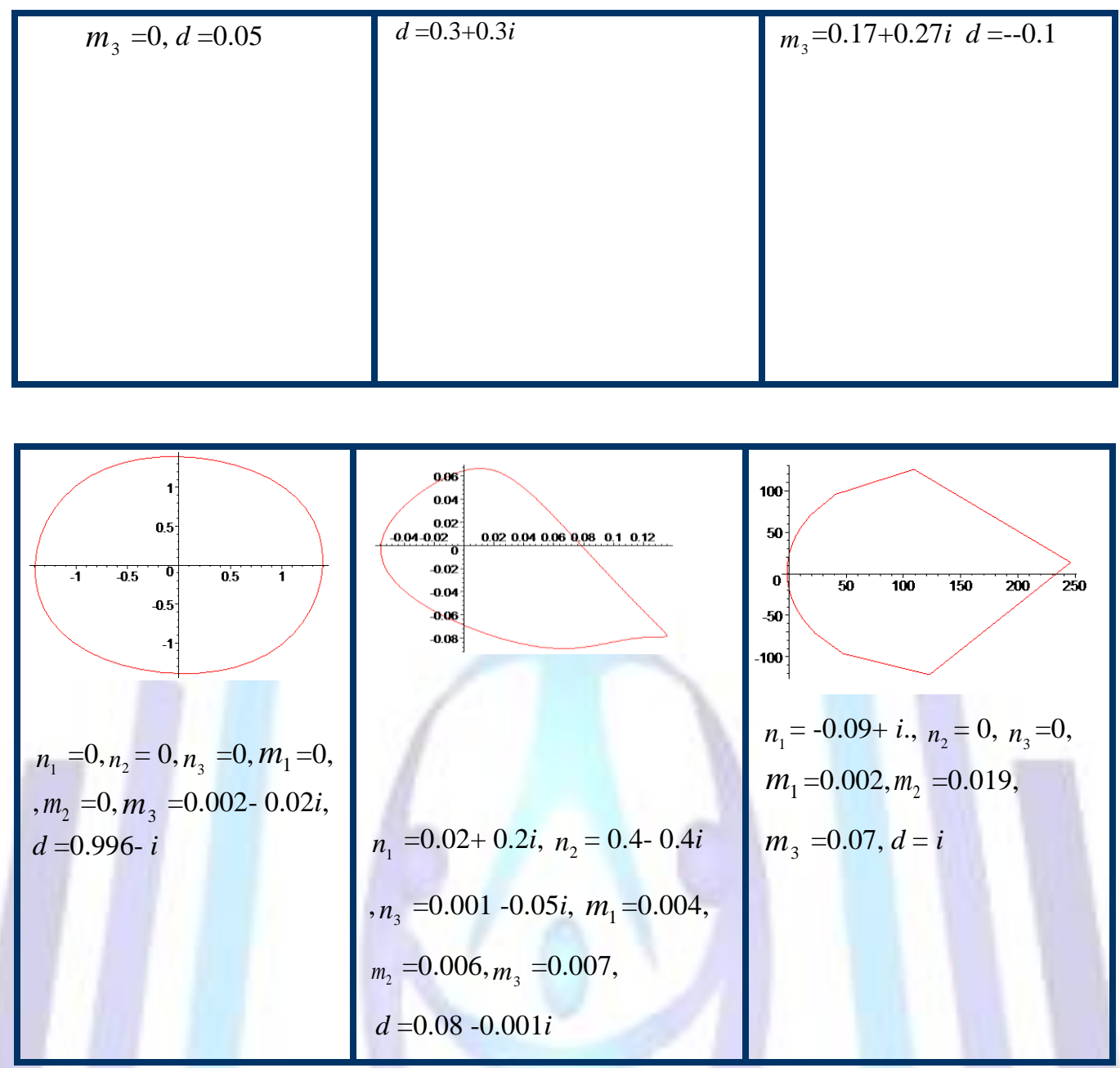

Fig(1) The different shapes of the rational mapping (6)

\section{4- Method of Solution:}

In this section ,we use the complex variable method to obtain the two complex functions (Goursat functions) $\phi(\zeta)$ and $\psi(\zeta)$. Moreover, the three stress components $\sigma_{x x}, \sigma_{y y}, \sigma_{x y}$ will be completely determined.

\section{1- The Components of Stresses:}

It is known that, the components of stresses are given by, see[4]

$$
\begin{gathered}
\sigma_{x x}+\sigma_{y y}=4 \operatorname{Re}\left\{\phi^{\prime}(z)\right\} \\
\sigma_{y y}-\sigma_{x x}+i \sigma_{x y}=2\left\{\bar{z} \phi^{\prime \prime}(z)+\psi^{\prime}(z)\right\}
\end{gathered}
$$

Hence, we have

$$
\begin{array}{cc}
\sigma_{y y}=\operatorname{Re}\left\{2 \phi^{\prime}(z)+M(z, \bar{z})\right\}, \quad M(z, \bar{z})=\bar{z} \phi^{\prime \prime}(z)+\psi^{\prime}(z) \\
\sigma_{x x}=\operatorname{Re}\left\{2 \phi^{\prime}(z)-M(z, \bar{z})\right\}, \quad M(z, \bar{z})=\bar{z} \phi^{\prime \prime}(z)+\psi^{\prime}(z)
\end{array}
$$

and

$$
\sigma_{x y}=2 \operatorname{Im}\left\{\bar{z} \phi^{\prime \prime}(z)+\psi^{\prime}(z)\right\}=2 \operatorname{Im}\{M(z, \bar{z})\}
$$

\section{2- Goursat functions:}


To obtain the tow complex potential functions (Goursat functions) by using the conformal mapping (6) in the boundary condition (2)We write the expression $\frac{w(\zeta)}{w^{\prime}\left(\zeta^{-1}\right)}$ in the form ,

$$
\frac{w(\zeta)}{w^{\prime}\left(\zeta^{-1}\right)}=\alpha(\zeta)+\beta\left(\zeta^{-1}\right)
$$

where,

$$
\alpha(\zeta)=\sum_{j=1}^{3} \frac{h_{j}}{\zeta-n_{j}}
$$

$\beta\left(\zeta^{-1}\right)$ is a regular function for $|\zeta|>1, \frac{w(\zeta)}{w^{\prime}\left(\zeta^{-1}\right)}$ has a singularity at $\zeta=n_{1}, \zeta=n_{2}$ and $\zeta=n_{3}$.

$$
\begin{gathered}
h_{1}=\frac{1}{\left(n_{1}-n_{2}\right)\left(n_{1}-n_{3}\right)} \cdot L_{1} \\
h_{2}=\frac{1}{\left(n_{2}-n_{1}\right)\left(n_{2}-n_{3}\right)} \cdot L_{2} \\
h_{3}=\frac{1}{\left(n_{3}-n_{1}\right)\left(n_{3}-n_{2}\right)} \cdot L_{3} \\
L_{j}=\frac{\left(d n_{j}{ }^{4}+m_{1} n_{j}{ }^{2}+m_{2} n_{j}+m_{3}\right)\left(1-\overline{n_{1}} n_{j}\right)^{2}\left(1-\overline{n_{2}} n_{j}\right)^{2}\left(1-\overline{n_{3}} n_{j}\right)^{2}}{\left\{\left(4 \bar{d}+2 \overline{m_{1}} n_{j}{ }^{2}+\overline{m_{2}} n_{j}\right)\left(1-\overline{n_{1}} n_{j}\right)\left(1-\overline{n_{2}} n_{j}\right)\left(1-\overline{n_{3}} n_{j}\right)-\left(\bar{d}+\overline{m_{1}} n_{j}{ }^{2}+\overline{m_{2}} n_{j}{ }^{3}+\overline{m_{3}} n_{j}{ }^{4}\right)\right.} \\
\left.\left[\left(1-\overline{n_{1}} n_{j}\right)\left(1-\overline{n_{2}} n_{j}\right)+\left(1-\overline{n_{1}} n_{j}\right)\left(1-\overline{n_{3}} n_{j}\right)+\left(1-\overline{n_{2}} n_{j}\right)\left(1-\overline{n_{3}} n_{j}\right)\right]\right\}, j=1,2,3
\end{gathered}
$$

Using Eq.(3) and Eq.(4) in Eq.(1), we get

$$
k \phi(\sigma)-\alpha(\sigma) \overline{\phi^{\prime}(\sigma)}-\overline{\psi_{*}(\sigma)}=G(\sigma)
$$

where

$$
\begin{gathered}
\psi_{*}(\sigma)=\psi(\sigma)+\beta(\sigma) \phi^{\prime}(\sigma) \\
G(\sigma)=F(\sigma)-c d k \Gamma \sigma+\frac{c \bar{d} \overline{\Gamma^{*}}}{\sigma}+N(\sigma) \alpha(\sigma)+N(\sigma) \overline{\beta(\sigma)} \\
N(\sigma)=\left[c \bar{d} \bar{\Gamma}-\frac{\sigma(X-i Y)}{2 \pi(1+\chi)}\right], \quad F(\sigma)=f(c w(\sigma))=f(t) .
\end{gathered}
$$

Assume that the function $F(\sigma)$ with it's derivatives must satisfy the Hölder condition. our aim is to determine the functions $\phi(\zeta)$ and $\psi(\zeta)$ for the various boundary value problems. For this multiply both sides of Eq. (19) by $\frac{d \sigma}{2 \pi i(\sigma-\zeta)}$ , where $\zeta$ is any point in the interior of $\gamma$ and integral over the circle, we obtain

$$
\frac{k}{2 \pi i} \int_{\gamma} \frac{\phi(\sigma)}{\sigma-\zeta} d \sigma-\frac{1}{2 \pi i} \int_{\gamma} \frac{\alpha(\sigma) \overline{\phi^{\prime}}(\sigma)}{\sigma-\zeta} d \sigma-\frac{1}{2 \pi i} \int_{\gamma} \frac{\overline{\psi_{*}}(\sigma)}{\sigma-\zeta} d \sigma=\frac{1}{2 \pi i} \int_{\gamma} \frac{G(\sigma)}{\sigma-\zeta} d \sigma
$$

Using Eqs.(20)-(22) in Eq.(23) then applying the properties of Cauchy integral, to have 


$$
\frac{k}{2 \pi i} \int_{\gamma} \frac{\phi(\sigma)}{\sigma-\zeta} d \sigma=-k \phi(\zeta)
$$

and

$$
\begin{aligned}
& \frac{1}{2 \pi i} \int_{\gamma} \frac{\alpha(\sigma) \overline{\phi^{\prime}}(\sigma)}{\sigma-\zeta} d \sigma=c \sum_{j=1}^{3} \frac{h_{j} b_{j}}{n_{j}-\zeta} \\
& \frac{1}{2 \pi i} \int_{\gamma} \frac{N(\sigma) \alpha(\sigma)}{(\sigma-\zeta)} d \sigma=\sum_{j=1}^{3} \frac{N\left(n_{j}\right) h_{j}}{n_{j}-\zeta}
\end{aligned}
$$

where $b_{j}, j=1,2,3$ are complex constants which can be determined.

Also,

$$
\frac{1}{2 \pi i} \int_{\gamma} \frac{G(\sigma)}{(\sigma-\zeta)} d \sigma=A(\zeta)-\frac{c \overline{d \Gamma^{*}}}{\zeta}+\sum_{j=1}^{3} \frac{N\left(n_{j}\right) h_{j}}{n_{j}-\zeta}
$$

where,

$$
A(\zeta)=\frac{1}{2 \pi i} \int_{\gamma} \frac{F(\sigma)}{(\sigma-\zeta)} d \sigma
$$

From the above ,Eq.(23) becomes

$$
-k \phi(\zeta)=A(\zeta)+\sum_{j=1}^{3} \frac{h_{j}}{n_{j}-\zeta}\left(c b_{j}+N\left(n_{j}\right)\right)-\frac{c \bar{d} \overline{\Gamma^{*}}}{\zeta}
$$

Determining Eq.(29) with respect to $\zeta$ and using the result in Eq.(26), we obtain

$$
c k b_{j}+\overline{A^{\prime}\left(n_{j}\right)}+c d \Gamma^{*} n_{j}^{2}+v_{j} \overline{h_{j}}\left(c \overline{b_{j}}+\overline{N\left(n_{j}\right)}\right)=0
$$

where,

$$
v_{j}=\frac{n_{j}^{2}}{\left(1-n_{j} \overline{n_{j}}\right)^{2}} \quad, j=1,2,3
$$

Hence, we have

$$
b_{j}=\frac{k E_{j}-v_{j} \overline{h_{j}} \overline{E_{j}}}{c\left(k^{2}-v_{j} \overline{v_{j}} h_{j} \overline{h_{j}}\right)} \quad, j=1,2,3
$$

where,

$$
E_{j}=-\overline{A^{\prime}\left(n_{j}\right)}-c d \Gamma^{*} n_{j}{ }^{2}-v_{j} \overline{h_{j}} \overline{N\left(n_{j}\right)}
$$

To obtain the complex function $\psi(\zeta)$ we have form Eq.(20) after substituting the expression of $\psi_{*}(\sigma)$ and $G(\sigma)$, and taking the complex conjugate of the resulting equation after using the expression of $\overline{\beta(\sigma)}$ to yields,

$$
\psi(\sigma)=-\overline{F(\sigma)}+c k \bar{d} \bar{\Gamma} \sigma^{-1}-c d \Gamma^{*} \sigma+k \overline{\phi(\sigma)}-\overline{\alpha(\sigma)} \phi_{*}(\sigma)-\frac{\overline{w(\sigma)}}{w^{\prime}(\sigma)} \phi_{*}(\sigma)+\sum_{j=1}^{3} \frac{\overline{h_{j}} \sigma}{\left(1-\overline{n_{j}} \sigma\right)} \phi_{*}(\sigma)
$$

where,

$$
\phi_{*}(\sigma)=\phi^{\prime}(\sigma)+\overline{N(\sigma)} \quad, \overline{N(\sigma)}=\left[c d \Gamma-\frac{\sigma^{-1}(X+i Y)}{2 \pi(1+\chi)}\right]
$$


and calculate sum residue, we obtain Multiplying both sides of Eq.(34) by $\frac{1}{2 \pi i(\sigma-\zeta)}$, where $\zeta$ is any point in the interior of $\gamma$ and integrating over the circle, then using the properties of Cauchy's integral and calculating the sum residue ,we obtain

$$
\psi(\zeta)=c k \bar{d} \bar{\Gamma} \zeta^{-1}-\frac{\overline{w(\zeta)}}{w^{\prime}(\zeta)} \phi_{*}(\zeta)+\sum_{j=1}^{3} \frac{\overline{h_{j}} \zeta}{\left(1-\overline{n_{j}} \zeta\right)} \phi_{*}\left(n_{j}{ }^{-1}\right)+B(\zeta)-B
$$

where,

$$
B(\zeta)=\frac{1}{2 \pi i} \int_{\gamma} \frac{\overline{F(\sigma)}}{(\sigma-\zeta)} d \sigma
$$

and

$$
B=\frac{1}{2 \pi i} \int_{\gamma} \frac{\overline{F(\sigma)}}{\sigma} d \sigma
$$

\section{5- Special cases:}

Here, we discuss the following:

1) By considering the reality of the constants of the mapping (6), the Goursat functions, when $d=1, m_{2}, m_{3}, n_{3}=0$ are agree with work of England [1]

$$
z=c \frac{\left(\zeta+m_{1} \zeta^{-1}\right)}{\left(1-n_{1} \zeta^{-1}\right)\left(1-n_{2} \zeta^{-1}\right)}
$$

2) When $d=1, m_{3}=n_{j}=0, j=1,2,3$, the transformation mapping (6) becomes

$$
z=c\left(\zeta+m_{1} \zeta^{-1}+m_{2} \zeta^{-2}\right) \quad, 0 \leq m_{1} \leq 1,0 \leq m_{2} \leq 1
$$

The Goursat functions, in this case, of the two formulas (29) and (36) agree with the all results of Abdou and Khamis [12].

3) For $m_{j}=0,1 \leq j \leq 3$, we get the mapping function represent of the hole is an ellipse, see Fig. (2)

$$
\begin{gathered}
z=c \frac{d \zeta}{\left(1-n_{1} \zeta^{-1}\right)\left(1-n_{2} \zeta^{-1}\right)\left(1-n_{3} \zeta^{-1}\right)} \\
h_{1}=\frac{1}{\left(n_{1}-n_{2}\right)\left(n_{1}-n_{3}\right)} \cdot M_{1} \\
h_{2}=\frac{1}{\left(n_{2}-n_{1}\right)\left(n_{2}-n_{3}\right)} \cdot M_{2}
\end{gathered}
$$

$$
h_{3}=\frac{1}{\left(n_{3}-n_{1}\right)\left(n_{3}-n_{2}\right)} \cdot M_{3}
$$

$$
M_{j}=\frac{d n_{j}{ }^{4}\left(1-\overline{n_{1}} n_{j}\right)^{2}\left(1-\overline{n_{2}} n_{j}\right)^{2}\left(1-\overline{n_{3}} n_{j}\right)^{2}}{\left\{4 \bar{d}\left(1-\overline{n_{1}} n_{j}\right)\left(1-\overline{n_{2}} n_{j}\right)\left(1-\overline{n_{3}} n_{j}\right)-\bar{d}\left(\left(1-\overline{n_{1}} n_{j}\right)\left(1-\overline{n_{2}} n_{j}\right)+\left(1-\overline{n_{1}} n_{j}\right)\left(1-\overline{n_{3}} n_{j}\right)+\left(1-\overline{n_{2}} n_{j}\right)\left(1-\overline{n_{3}} n_{j}\right)\right)\right\}}, j=1,2,3 \text { (45) }
$$

the Goursat functions become

$$
-k \phi(\zeta)=A(\zeta)+\sum_{j=1}^{3} \frac{h_{j}}{n_{j}-\zeta}\left(c b_{j}+N\left(n_{j}\right)\right)-\frac{c \bar{d} \overline{\Gamma^{*}}}{\zeta}
$$




$$
\psi(\zeta)=c k \bar{d} \bar{\Gamma} \zeta^{-1}-\frac{\overline{w(\zeta)}}{w^{\prime}(\zeta)} \phi_{*}(\zeta)+\sum_{j=1}^{3} \frac{\overline{h_{j}} \zeta}{\left(1-\overline{n_{j}} \zeta\right)} \phi_{*}\left(n_{j}{ }^{-1}\right)+B(\zeta)-B
$$

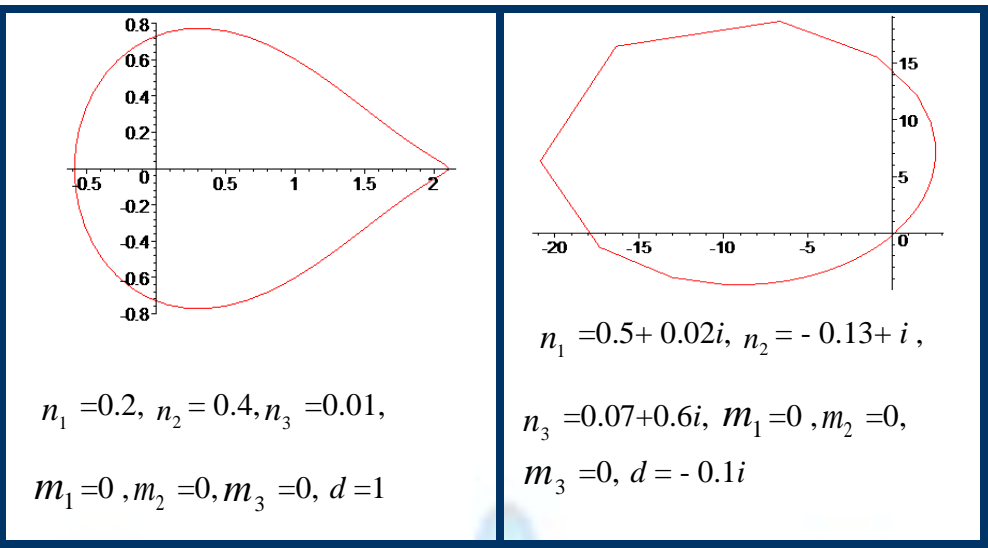

Fig.(2) The different shapes of the rational mapping for special cases

4) When $m_{j}=n_{j}=0, j=1,2$, the transformation mapping (6) becomes

$$
z=c \frac{d \zeta+m_{1} \zeta^{-1}}{1-n_{1} \zeta^{-1}} \quad, 0 \leq m_{1} \leq 1, n_{1} \neq 0
$$

The Goursat functions, in this case, of the two formulas (29) and (36) agree with the all results of Abdou and Asseri [18] and M.A. Abdou1 and A. R. Jan [19] at different discussed.

\section{6-Some applications:}

In this section, we assume different values of the given functions in the first or second fundamental boundary value problems. Then ,we obtain the expression of Goursat functions. After that, the components of stresses can be calculated directly.

\section{Application 1:}

Curvilinear hole for an infinite layer subjected to uniform tensile stress For $k=-1, \Gamma=\frac{p}{4}, \Gamma^{*}=-\frac{1}{2} p e^{-2 i \theta}$ and $X=Y=f=0,0 \leq \theta \leq 2 \pi$, we have the case of infinite plate stretched at infinity by the application of a uniform tensile stress of intensity $p$, making an angle $\theta$ with the x-axis. The plate weakened by the curvilinear hole $C$ which is free form stresses, see $\quad$ Figs.(3-4) $\left(d=0.1+i, n_{1}=0.02+0.2 i, n_{2}=0.04-0.4 i, n_{3}=0.05+0.05 i\right.$ $\left.m_{1}=0.004, m_{2}=0.007, m_{3}=0.002, c=2, p=0.25\right)$. Then the functions in (29) and (36) becomes

$$
\begin{gathered}
f=0 \Rightarrow A(\zeta)=0 \\
N\left(n_{j}\right)=\left[c \bar{d} \bar{\Gamma}-\frac{n_{i}(X-i Y)}{2 \pi(1+\chi)}\right]=\frac{c p \bar{d}}{4} \quad, j=1,2,3 \\
E_{j}=\frac{2 c d n_{j}^{2} p e^{-2 i \theta}-v_{j} \overline{h_{j}} c p d}{4} \overline{E_{j}}=\frac{2 c \bar{d} \bar{n}_{j}^{2} p e^{2 i \theta}-\overline{v_{j}} h_{j} c p \bar{d}}{4}, j=1,2,3 \\
c b_{j}=\frac{-E_{j}-v_{j} \overline{h_{j}} \overline{E_{j}}}{\left(1-v_{j} \bar{v}_{j} h_{j} \overline{h_{j}}\right)}, j=1,2,3 \\
\phi(\zeta)=\frac{c p \bar{d} e^{2 i \theta}}{2 \zeta}+\sum_{j=1}^{3} \frac{h_{j}}{\left(n_{j}-\zeta\right)}\left(c b_{j}+N\left(n_{j}\right)\right) \\
\psi(\zeta)=\frac{-c p \bar{d}}{4 \zeta}-\frac{w\left(\zeta^{-1}\right)}{w^{\prime}(\zeta)} \phi_{*}(\zeta)+\sum_{j=1}^{3} \frac{\overline{h_{j}} \zeta}{\left(1-\overline{n_{j}} \zeta\right)} \phi_{*}\left(n_{j}{ }^{-1}\right)
\end{gathered}
$$




$$
\phi_{*}(\zeta)=\phi^{\prime}(\zeta)+\frac{c p d}{4}
$$

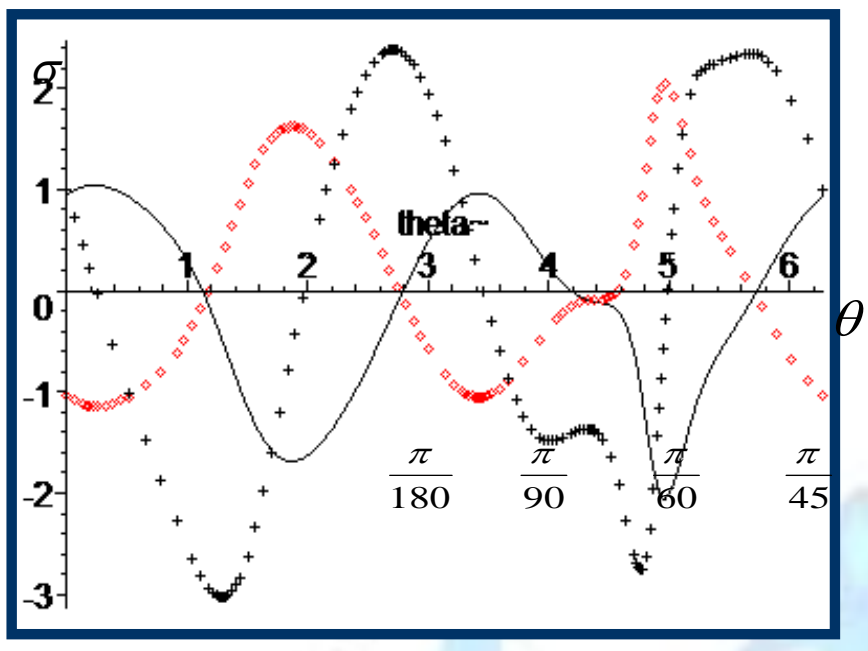

$$
\begin{aligned}
& \max \sigma_{x x} \text { at } \theta \approx \frac{\pi}{36} \cdot \\
& \min \sigma_{x x} \text { at } \theta \text { between }\left(0, \frac{\pi}{180}\right),\left(\frac{\pi}{60}, \frac{\pi}{45}\right) \\
& \max \sigma_{y y} \text { at } \theta \text { between }\left(0, \frac{\pi}{180}\right),\left(\frac{\pi}{60}, \frac{\pi}{45}\right) \\
& \min \sigma_{y y} \text { at } \theta \approx \frac{\pi}{36} \cdot \\
& \max \sigma_{x y} \text { at } \theta \approx \frac{\pi}{60} \text { and between }\left(\frac{\pi}{36}, \frac{\pi}{30}\right) \\
& \min \sigma_{x y} \text { at } \theta \text { between }\left(\frac{\pi}{90}, \frac{\pi}{180}\right) \\
& \sigma_{x y}^{+++} \sigma_{y y}-\ldots . . \sigma_{x x}
\end{aligned}
$$

Fig.(3): The relation between components of stresses and the angle made on the $x$-axis

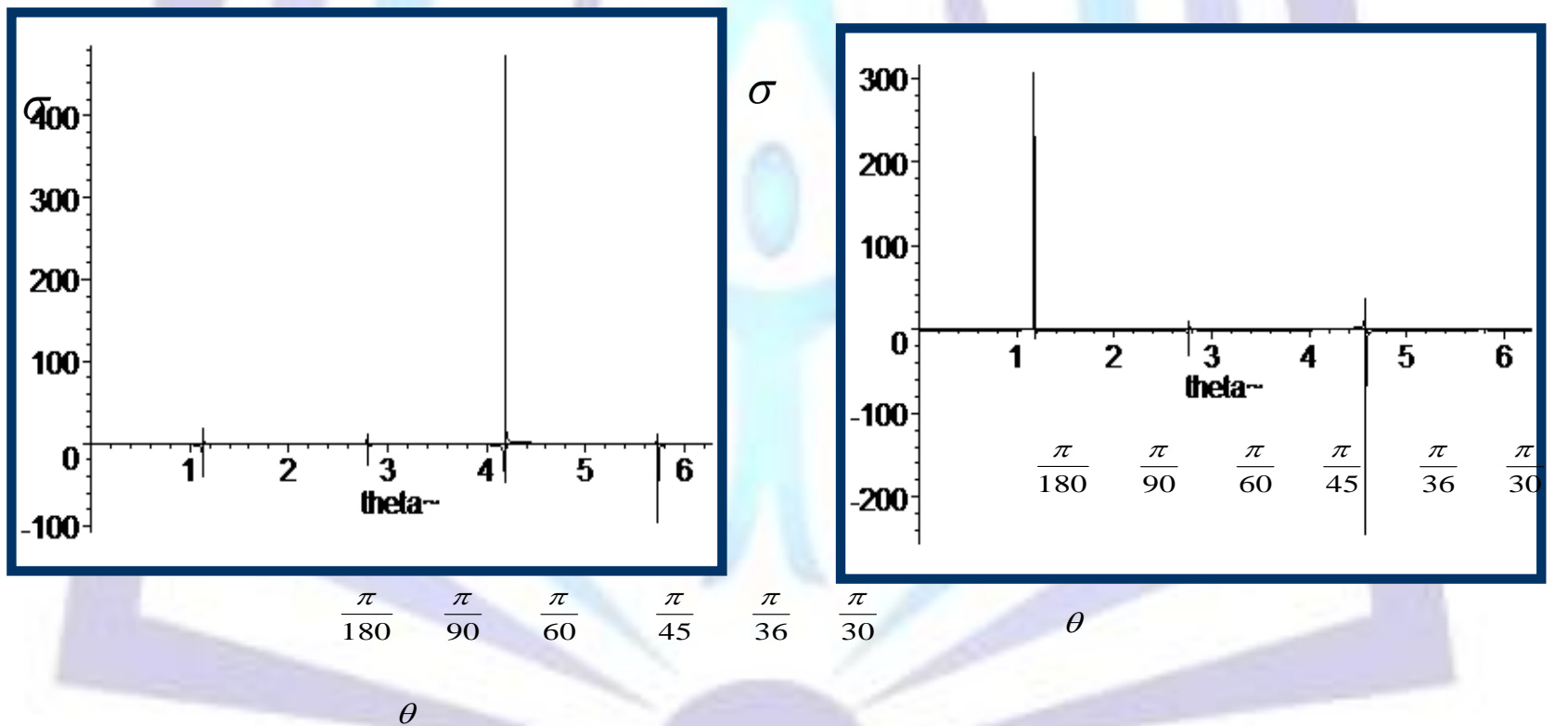

Fig(4): $\max _{\frac{\sigma_{x x}}{\sigma_{y y}}}$ at $\theta$ between $\frac{\pi}{36}, \frac{\pi}{45}, \min \frac{\sigma_{x x}}{\sigma_{y y}}$ at $\theta$ between $\frac{\pi}{36}, \frac{\pi}{30}$ $\max _{\frac{\sigma_{y y}}{\sigma_{x x}}}$ at $\theta$ between $\frac{\pi}{90}, \frac{\pi}{180}, \min \frac{\sigma_{y y}}{\sigma_{x x}}$ at $\theta$ between $\frac{\pi}{45}, \frac{\pi}{36}$

\section{Application 2:}

For $k=-1, \Gamma=\Gamma^{*}=X=Y=0$ and $f=P t$, where $P$ is a real constant see Figs.(5-

$6),\left(d=0.1+i, n_{1}=0.02+0.2 i, n_{2}=0.04-0.4 i, n_{3}=0.05+0.05 i, c=2, p=0.25\right.$

$\left.m_{1}=0.004, m_{2}=0.007, m_{3}=0.002\right)$. Then the functions in (29) and (36) becomes

$$
f=P t=c P \frac{d \sigma^{4}+m_{1} \sigma^{2}+m_{2} \sigma+m_{3}}{\left(\sigma-n_{1}\right)\left(\sigma-n_{2}\right)\left(\sigma-n_{3}\right)}
$$




$$
\begin{aligned}
& \bar{f}=c P \frac{\bar{d}+\overline{m_{1}} \sigma^{2}+\overline{m_{2}} \sigma^{3}+\overline{m_{3}} \sigma^{4}}{\sigma\left(1-n_{1} \sigma\right)\left(1-\overline{n_{2}} \sigma\right)\left(1-\overline{n_{3}} \sigma\right)} \\
& N\left(n_{i}\right)=0 \\
& A(\zeta)=c P \sum_{j=1}^{3}\left[\frac{d n_{j}{ }^{4}+m_{1} n_{j}{ }^{2}+m_{2} n_{j}+m_{3}}{\left(n_{j}-n_{j+1}\right)\left(n_{j}-n_{j+2}\right)\left(n_{j}-\zeta\right)}\right] \\
& \overline{A^{\prime}\left(n_{j}\right)}=c P \sum_{j=1}^{3}\left[\frac{n_{j}^{2}\left(\bar{d} \bar{n}_{j}^{4}+\bar{m}_{1}{\overline{n_{j}}}^{2}+\overline{m_{2}} \bar{n}_{j}+\bar{m}_{3}\right)}{\left(\overline{n_{j}}-\overline{n_{j+1}}\right)\left(\overline{n_{j}}-\overline{n_{j+2}}\right)\left(\overline{n_{j}} n_{j}-1\right)^{2}}\right] \\
& E_{j}=-\overline{A^{\prime}\left(n_{j}\right)}, \bar{E}_{j}=A^{\prime}\left(n_{j}\right) \\
& c b_{j}=\frac{-E_{j}-v_{j} \overline{h_{j}} \overline{E_{j}}}{\left(1-v_{j} \overline{v_{j}} h_{j} \overline{h_{j}}\right)} \quad, j=1,2,3 \\
& \phi(\zeta)=A(\zeta)+\sum_{j=1}^{3} \frac{h_{j}}{n_{j}-\zeta} c b_{j} \\
& \psi(\zeta)=-c P \sum_{j=1}^{3}\left(\overline{n_{j}}+\zeta^{-1}\right) \bar{d}-\frac{w\left(\zeta^{-1}\right)}{w^{\prime}(\zeta)} \phi_{*}(\zeta)+\sum_{j=1}^{3} \frac{\overline{h_{j}} \zeta}{\left(1-\overline{n_{j}} \zeta\right)} \phi_{*}\left(n_{j}^{-1}\right)
\end{aligned}
$$

where

$$
\begin{aligned}
& B(\zeta)=\frac{1}{2 \pi i} \int_{\gamma} \frac{\overline{F(\sigma)}}{(\sigma-\zeta)} d \sigma=-\frac{c P \bar{d}}{\zeta} \\
& B=\frac{1}{2 \pi i} \int_{\gamma} \frac{\overline{F(\sigma)}}{\sigma} d \sigma=c P \sum_{j=1}^{3} \overline{n_{j}} \bar{d}, \quad \phi_{*}(\zeta)=\phi^{\prime}(\zeta) .
\end{aligned}
$$

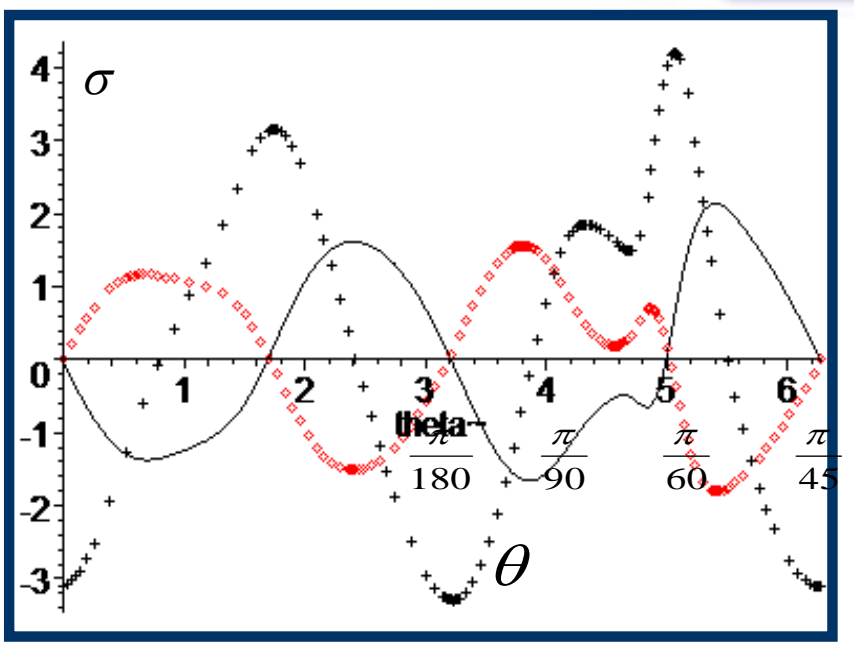

$$
\begin{aligned}
& \max \sigma_{x x} \text { at } \theta \approx \frac{\pi}{45} . \\
& \min \sigma_{x x} \text { at } \theta \text { between } \frac{\pi}{36}, \frac{\pi}{30} . \\
& \max \sigma_{y y} \text { at } \theta \text { between } \frac{\pi}{36}, \frac{\pi}{30} \\
& \min \sigma_{y y} \text { at } \theta \approx \frac{\pi}{45} \cdot \\
& \max \sigma_{x y} \text { at } \theta \approx \frac{\pi}{36} \cdot \\
& \min \sigma_{x y} \text { at } \theta \approx 0, \frac{\pi}{60}, \frac{\pi}{30} . \\
& \sigma_{x y}+++\sigma_{y y}-\cdots \cdot \sigma_{x x}
\end{aligned}
$$


Fig.(5): The relation between components of stresses and the angle made on the $x$-axis.

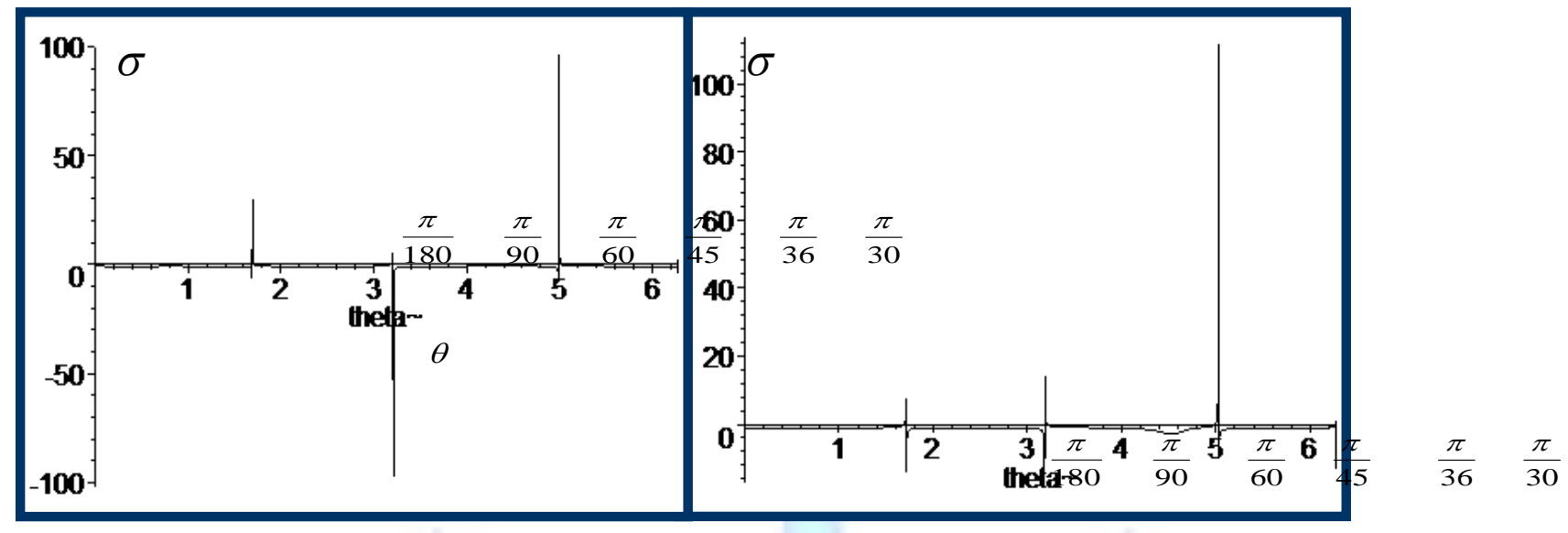

Fig(6): $\max _{\frac{\sigma_{x x}}{\sigma_{y y}}}$ at $\theta \approx \frac{\pi}{36},, \min \frac{\sigma_{x x}}{\sigma_{y y}}$ at $\theta \approx \frac{\pi}{60}$.

$\max \frac{\sigma_{y y}}{\sigma_{x x}}$ at $\theta \approx \frac{\pi}{36}, \min \frac{\sigma_{y y}}{\sigma_{x x}}$ at $\theta \approx \frac{\pi}{60}$

\section{APPLICATION 3:}

For

$$
k=\chi, \Gamma=\frac{p}{4}, \Gamma^{*}=-\frac{1}{2} p e^{-2 i \theta}, X=Y=0, f=2 \mu i t,
$$

$\left(d=0.1+i, n_{1}=0.02+0.2 i, n_{2}=0.04-0.4 i, n_{3}=0.05+0.05 i, m_{1}=0.004, m_{2}=0.007, m_{3}=0.002, c=2, p=0.25\right)$

. Then the functions in (29) and (36) becomes

$$
\begin{aligned}
& N\left(n_{j}\right)=\frac{c p \bar{d}}{4} \\
& A(\zeta)=2 c \mu i \sum_{j=1}^{3}\left[\frac{d n_{j}^{4}+m_{1} n_{j}^{2}+m_{2} n_{j}+m_{3}}{\left(n_{j}-n_{j+1}\right)\left(n_{j}-n_{j+2}\right)\left(n_{j}-\zeta\right)}\right] \\
& \overline{A^{\prime}\left(n_{j}\right)}=2 c \mu i \sum_{j=1}^{3}\left[\frac{n_{j}^{2}\left(\bar{d}{\overline{n_{j}}}^{4}+\bar{m}_{1}{\overline{n_{j}}}^{2}+\bar{m}_{2} \bar{n}_{j}+\bar{m}_{3}\right)}{\left(\overline{n_{j}}-\overline{n_{j+1}}\right)\left(\overline{n_{j}}-\overline{n_{j+2}}\right)\left(\overline{n_{j}} n_{j}-1\right)^{2}}\right] \\
& E_{j}=-\overline{A^{\prime}\left(n_{j}\right)}+\frac{2 c d n_{j}{ }^{2} p e^{-2 i \theta}-v_{j} \overline{h_{j}} c p d}{4} \quad, j=1,2,3 \\
& E_{j}=-A^{\prime}\left(n_{j}\right)+\frac{2 c \bar{d} \overline{n_{j}^{2}} p e^{2 i \theta}-\overline{v_{j}} h_{j} c p \bar{d}}{4} \quad, j=1,2,3 \\
& c b_{j}=\frac{\chi E_{j}-v_{j} h_{j} \overline{E_{j}}}{\left(1-v_{j}^{2} h_{j}^{2}\right)} \quad, j=1,2,3
\end{aligned}
$$




$$
\begin{gathered}
-\chi \phi(\zeta)=A(\zeta)+\frac{c p \bar{d} e^{2 i \theta}}{2 \zeta}+\sum_{j=1}^{3} \frac{h_{j}}{\left(n_{j}-\zeta\right)}\left(c b_{j}+N\left(n_{j}\right)\right) \\
\psi(\zeta)=c \bar{d} \chi \frac{p}{4}-2 c \mu i \sum_{j=1}^{3}\left(\overline{n_{j}}+\zeta^{-1}\right) \bar{d}-\frac{w\left(\zeta^{-1}\right)}{w^{\prime}(\zeta)} \phi_{*}(\zeta)+\sum_{j=1}^{3} \frac{\overline{h_{j}} \zeta}{\left(1-\overline{n_{j}} \zeta\right)} \phi_{*}\left(n_{j}^{-1}\right)
\end{gathered}
$$

where

$$
\begin{aligned}
& B(\zeta)=\frac{1}{2 \pi i} \int_{\gamma} \frac{\overline{F(\sigma)}}{(\sigma-\zeta)} d \sigma=-2 c \mu i \bar{d} \zeta^{-1} \\
& B=\frac{1}{2 \pi i} \int_{\gamma} \frac{\overline{F(\sigma)}}{\sigma} d \sigma=2 c \mu i \sum_{j=1}^{3} \overline{n_{j}} \bar{d}, \quad \phi_{*}(\zeta)=\phi^{\prime}(\zeta)+\frac{c p d}{4} .
\end{aligned}
$$

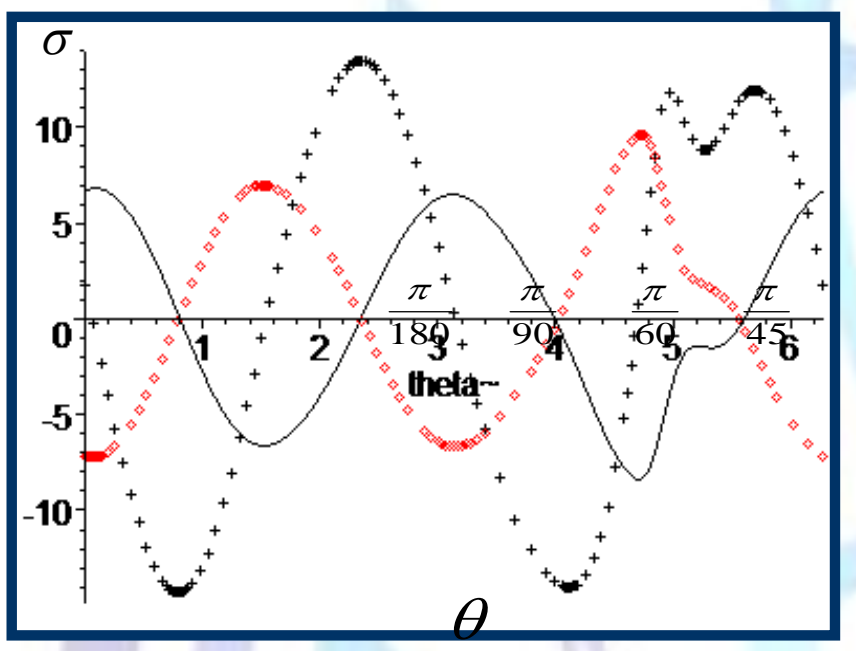

$\max \sigma_{x x}$ at $\theta$ between $_{\frac{\pi}{36}}, \frac{\pi}{45}$ $\min \sigma_{x x}$ at $\theta \approx 0, \frac{\pi}{60}, \frac{\pi}{30}$ $\max \sigma_{y y}$ at $\theta \approx 0, \frac{\pi}{60}, \frac{\pi}{30}$ $\min \sigma_{y y}$ at $\theta$ between $\frac{\pi}{36}, \frac{\pi}{45}$ $\max \sigma_{x y}$ at $\theta$ between $\frac{\pi}{90}, \frac{\pi}{60}$ $\min \sigma_{x y}$ at $\theta$ between $0, \frac{\pi}{180}$

$\sigma_{x y}^{+++} \sigma_{y y}-\cdots \cdot \sigma_{x x}$

Fig.(7): The relation between components of stresses and the angle made on the $\mathrm{x}$-axis.

$\sigma$

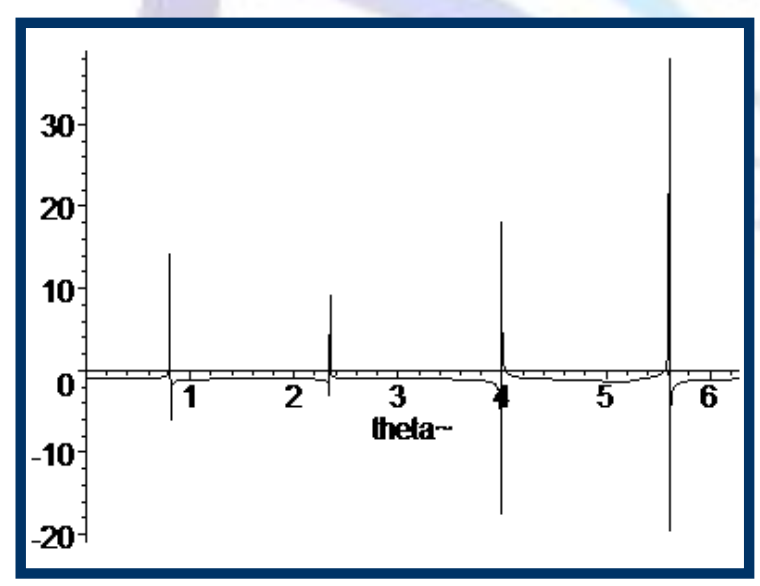

$\sigma$

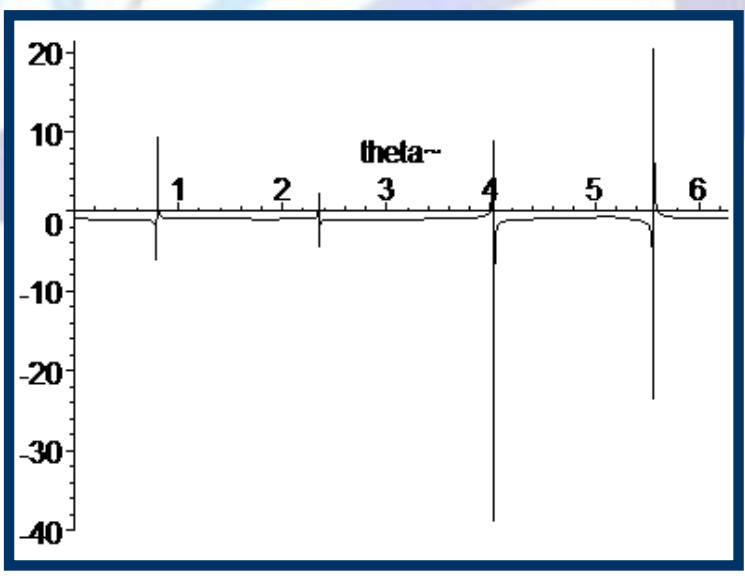

$\theta$

$\frac{\pi}{180} \quad \frac{\pi}{90} \quad \frac{\pi}{60} \quad \frac{\pi}{45} \quad \frac{\pi}{36} \quad \frac{\pi}{30} \quad \frac{\pi}{180} \quad \frac{\pi}{90} \quad \frac{\pi}{60} \quad \frac{\pi}{45} \quad \frac{\pi}{36} \quad \frac{\pi}{30}$ 
Fig(8): $\max _{\frac{\sigma_{x x}}{\sigma_{y y}}}$ at $\theta$ between $\frac{\pi}{3} \dot{\theta} \frac{\pi}{30} \min \frac{\sigma_{x x}}{\sigma_{y y}}$ at $\theta$ between $\frac{\pi}{36}, \frac{\pi}{30}$.

$$
\max \frac{\sigma_{y y}}{\sigma_{x x}} \text { at } \theta \text { between } \frac{\pi}{36}, \frac{\pi}{30} ., \min \frac{\sigma_{y y}}{\sigma_{x x}} \text { at } \theta \approx \frac{\pi}{45}
$$

\section{REFERENCE}

[1] A.H.England, Complex Variable Methods in Elasticity, London, New York, (1971).

[2] A.I.Kalandiya, Mathematical Theory of Elasticity, Mir Moscow, (1975).

[3] I.S.Sokolnikff, Mathematical Theory of Elasticity, New York,(1950).

[4] N.I.Muskhelishvili, Some Basic Problems of Mathematical Theory of Elasticity,

Noordhoff International, Leyden, he Netherlands, (1953).

[5] F.D.Gakhov, ., Boundary value problems, General publishing company, Ltd., Canada (1966).

[6] P. G. Ciarlet, M. H. Schultz, and R. S. Varga, Numerical methods of high-order accuracy for nonlinear boundary value problems I. One dimensional problem, Numer. Math., Vol. 9 , (1967).

[7] A. Zebib, A Chebyshev method for the solution of boundary value problems, J. Comput. Phys. Vol. 53, (1984).

[8] S.Saito, M.Yamamto, Boundary value problems of quasili-near ordinary differential systems on a finite interval. Math. Japon. Vol. $34,(1989)$.

[9] D. Colton, R. Kress, Integral Equation Methods in Scattering Theory, John Wiley, New York, (1983).

[10] M. A. Abdou, First and second fundamental problems for an elastic infinite plate with a curbilinear hole. Alex. Eng. J.Vol. 33, (1994).

[11] M. A. Abdou, Fundamental problems for infinite plate with a curvilinear hole having infinite poles. Appl. Math. Comput.,Vol.93, (2002).

[12] M. A. Abdou and A. K. Khamis, On a Problem of an Infinite Plate with a Curvilinear Hole Having Three Poles and Arbitrary Shape. Bulletin of Calcutta Mathematical Society, 92, 309-322,(2000).

[13] M. A. Abdou and S. J. Monaquel, Integro Differential Equation and Fundamental

Problems of an Infinite Plate with a Curvilinear Hole Having Strong Pole. Int. J. Contemp. Math. Sciences, Vol. 6(4), 199 208, (2011).

[14] F. S. Bayones and B. M. Alharbi, On a Problem of an Infinite Plate with a Curvilinear Hole inside the Unit Circle. Applied Mathematics, 6, 206-220, (2015).

[15] F.S.Bayones, Presence of Heat on an Infinite Plate with a Curvilinear Hole Having Two Poles. Journal of Modern Physics, 6, 837-853, (2015).

[16] F.S.Bayones, An Infinite Plate with a Curvilinear Hole Having Three Poles and Presence of Heat. Journal of Computational and Theoretical Nanoscience, 12, 1630-1640, (2015).

[17] H. Parkus, Thermo Elasticity. Spring-Verlag, Berlin, (1976).

[18] M. A. Abdou, and S.A. Asseri, Gaursat Functions for an Infinite Plate with a Generalized Curvilinear Hole in Zeta Plane. Applied Mathematics and Computation, 212, 23-36, (2009).

[19] M. A. Abdou and A. R. Jan, An Infinite Elastic Plate Weakened by a Generalized Curvilinear Hole and Goursat Functions. Applied Mathematics, 5, 728-743, (2014). 